\title{
FACTORES QUE AUMENTAN \\ LA EFICIENCIA DE LAS ENCUESTAS POSTALES ${ }^{1}$
}

\section{Vidal Díaz de Rada}

Universidad Pública de Navarra

E-mail: vidal@upna.es

\begin{abstract}
RESUMEN
El objetivo de este trabajo es analizar los factores que intervienen en la mejora de la calidad y la tasa de respuesta de las encuestas postales. Una de las razones principales que nos anima a realizar este trabajo es el hecho que la colaboración en las entrevistas cara a cara y en las telefónicas está bajando en los últimos años, mientras que las postales permanecen estables. Así, en este artículo se sintetizan los factores que inciden en la eficiencia de las encuestas postales, planteando como hipótesis principal que la tasa de respuesta y la calidad de los datos recogidos utilizando encuestas postales es semejante - y en ciertos momentos superior - a los recogidos por la encuesta personal y telefónica, con un coste económico notablemente inferior.
\end{abstract}

Presentamos un trabajo sobre la encuesta, uno de los procedimientos más populares de recogida de datos desde el gran desarrollo de la investigación de opinión y mercados en la sociedad americana de los años cuarenta. Pese a la gran importancia de la encuesta en la investigación social, llama la atención los escasos trabajos que se publican en nuestro país sobre los efectos, limitaciones, ventajas y nuevas innovaciones de esta técnica de recogida de datos. Este hecho es aún más sorprendente cuando se constata que el método de la encuesta está

Cuando estaba terminando este trabajo fallecía en un accidente de tráfico Carlos Mendive, compañero del Departamento de Sociología de la Universidad Pública de Navarra, al que me gustaría dedicar este artículo.

\section{Reis}


experimentando un gran desarrollo en los últimos años, destacando los avances producidos en la forma de administración: el tradicional cuestionario impreso en "papel" está perdiendo importancia por la aparición de nuevas innovaciones que realizan encuestas personales y telefónicas con la ayuda de un ordenador (CATI y CAPI), encuestas realizadas automáticamente mediante ordenador en las que el entrevistado pulsa los números del teléfono para seleccionar las opciones (TDE y VR), unido a otros sistemas como las encuestas por fax, correo electrónico, etc.

Este trabajo analiza los factores que inciden en la calidad de las encuestas que se realizan por correo, aunque también puede aplicarse a las denominadas «encuestas autorrellenadas». El elemento característico de estos cuestionarios es que son contestados por el entrevistado sin la "ayuda" del entrevistador, sin ninguna intervención por parte de una tercera persona ${ }^{2}$. Otro de los elementos definitorios es que el entrevistado debe introducir el cuestionario en un sobre y enviarlo al investigador. La encuesta postal es el método de recogida de datos que menos se utiliza en las investigaciones realizadas en nuestro país, tal y como se desprende del estudio "Industria de los Estudios de Mercado en España 1995". Según este trabajo, un 61 por 100 de los estudios cuantitativos utilizó encuestas personales, el 26 por 100 entrevistas telefónicas y sólo un 7 por 100 cuestionarios postales (Alós, 1996). Esta baja utilización no se produce únicamente en nuestro país, sino que es generalizable a otros contextos: la encuesta postal ha sido uno de los métodos de recogida de datos menos utilizados, como se puede comprobar tras analizar las referencias bibliográficas sobre este tipo de investigaciones realizadas en los últimos años. Entre las razones que han motivado esta escasa utilización, una de las más importantes es la baja tasa de respuesta conseguida por este medio de recogida de datos, unida a la baja calidad de los mismos. Diversos autores afirman que la tasa de respuesta de las encuestas postales no suele sobrepasar el 30 por 100 (Meyers y Grossen, 1974; Black y Champion, 1976), considerando por ello más que aceptable una respuesta del 50 por 100 (Bailey, 1978). Así, numerosos textos recomiendan utilizar encuestas postales en ocasiones muy particulares, y muy pocas veces se aconseja este método de recogida de datos para investigaciones que tienen como objeto de estudio la población general.

No obstante, en los últimos años el ámbito de las encuestas postales ha sufrido una gran transformación debido, entre otras razones, a la obra de D. A. Dillman y a los trabajos de sus colaboradores. Las mejoras en la tasa de respuesta y en la calidad de la misma producidas por el Total Desing MethodTDM de Dillman han modificado totalmente la «mala prensa" atribuida a las encuestas postales. Como pone de manifiesto Dillman tras analizar 50 investigaciones postales, la tasa media de respuesta de las encuestas postales ronda el 77 por 100 , llegando a alcanzar el 90 por 100 cuando se trata de grupos espe-

La situación ideal es que el entrevistado conteste el cuestionario a solas, aunque, como veremos más adelante, las condiciones ideales no siempre se convierten en situaciones reales. 
cializados (1983). El hecho que todo el trabajo de este autor se haya realizado en la sociedad americana, ¿podría significar que esta elevada tasa de respuesta es característica de una sociedad como la americana, y que será totalmente distinta en la sociedad europea? Este planteamiento fue adoptado como hipótesis de trabajo por Goyder (1982) en la investigación que realizó para probar si la tasa de respuesta de las encuestas postales en Europa era menor que en USA debido, fundamentalmente, a las diferencias culturales entre ambas sociedades. Los resultados de esta investigación, así como otras similares realizadas en diversos países europeos (De Leeuw, 1988 y 1996; Nederhof, 1983, 1985a y 1988), Japón (Jussaume y Yamada, 1990) y Australia (Armstrong y Lusk, 1987), han conseguido tasas de respuesta similares a las conseguidas por Dillman en USA, desechando la hipótesis de Goyder de que las tasas de respuesta en Europa son menores que en América. Tras una exhaustiva búsqueda no hemos encontrado ninguna investigación que utilice el método de Dillman en nuestro país. Por ello decidimos utilizar esta metodología en una investigación sobre hábitos de consumo realizada durante la primavera de 1998 en el Departamento de Sociología de la Universidad Pública de Navarra. La tasa media de respuesta de la población navarra fue del 62,4 por 100 , aspecto que nos lleva a rechazar la hipótesis de Goyder sobre las diferencias en las tasas de respuesta entre la sociedad americana y la europea.

El objetivo de este artículo es analizar los factores que intervienen en la mejora de la calidad y la tasa de respuesta de las encuestas postales. Una de las razones principales que nos anima a realizar este trabajo es el hecho, señalado por numerosos estudiosos, que la colaboración en las entrevistas cara a cara y en las telefónicas está bajando en los últimos años, mientras que las postales permanecen estables (Goyder, 1987; Sugiyama, 1992). Así, el fin de este trabajo es sintetizar qué factores inciden en la eficiencia de las encuestas postales, planteando como hipótesis principal que la tasa de respuesta y la calidad de los datos recogidos utilizando encuestas postales es semejante $-\mathrm{y}$ en ciertos momentos superior- a los recogidos por la encuesta personal y telefónica, con un coste económico notablemente inferior. Desde nuestro punto de vista, la escasa utilización de este tipo de encuestas en nuestro país no siempre se debe a la supuesta baja calidad de los datos recogidos, sino más bien al escaso conocimiento de las técnicas y recursos que son imprescindibles en el diseño de un estudio postal.

Un buen indicador de calidad es la Tasa de Respuesta, definida como el número de cuestionarios completos entre el número de cuestionarios enviados menos el número de no elegibles (direcciones incorrectas, incompletas, desconocidas, etc.) (Kviz, 1977). Existen otras definiciones más complejas ${ }^{3}$ de cali-

Número de preguntas no contestadas, efecto de "deseabilidad social" en las respuestas, velocidad en la respuesta, dobles respuestas en una pregunta de una única respuesta, lectura correcta de las instrucciones, número de preguntas donde se ha respondido la opción no contesta, etc. 
dad en la respuesta, por lo que adoptaremos la definición utilizada por cada autor en cada uno de sus trabajos, definición que será explicada en el texto o en una nota a pie de página. Algún lector podría criticar la escasa precisión de estas definiciones, pero es el propio diseño del trabajo el que nos obliga a ser cautos en las mismas. Como se ha expuesto anteriormente, el objeto de este trabajo es sintetizar las aportaciones realizadas en las publicaciones sobre el tema, y en ciertos momentos hay grandes diferencias en las definiciones adoptadas por cada autor: algunos trabajos no hacen ninguna referencia a la definición de tasa y calidad en la respuesta, mientras que otros "construyen" sus propios indicadores para medir la calidad de la respuesta. Así, en las situaciones en las que un autor utilice una definición, ésta será explicada en el texto o en una nota a pie de página.

Este trabajo está dividido en cuatro partes que sintetizan los distintos factores que aumentan la eficiencia de las encuestas postales. En primer lugar se exponen los distintos factores que influyen en la respuesta de las encuestas independientemente del método de administración. En la segunda parte se analizará la "peculiar" toma de contacto con los entrevistados propia de la encuesta postal; en la que apenas hay contacto verbal (personal) entre el investigador y los entrevistados, y los medios que ponen en relación a ambas partes son el sobre y la carta de presentación, el tipo de correo y el sobre de reenvío ${ }^{4}$. Diversos factores propios del cuestionario condicionan la respuesta, tal y como se podrá comprobar en la tercera parte del artículo. Antes de dar paso a la conclusión, en la cuarta parte se analizará el efecto de un factor más difícil de controlar como es la influencia de la institución que promueve la investigación. El artículo terminará exponiendo las principales conclusiones de todo lo expuesto y los campos de no aplicación de la encuesta postal.

Dentro de cada uno de estos apartados se expondrán los factores intervinientes según la proximidad de cada uno de ellos con el entrevistado: el entrevistado analiza en primer lugar el sobre y el tipo de correo utilizado y la personificación de la correspondencia. Posteriormente leerá la carta de presentación y, por último, utilizará el sello y el sobre de reenvío.

\section{FACTORES INFLUYENTES EN LA ACEPTACIÓN DE LAS ENCUESTAS}

En esta primera parte se analizarán factores que influyen en la colaboración de los entrevistados independientemente del medio de recogida de datos: el aumento del número de contactos antes y después del envío del primer cues-

El contacto verbal puede existir puesto que en la carta de presentación es usual que el investigador facilite un teléfono para solucionar las dudas, recelos, etc., de los entrevistados. Además, puede existir un precontacto o un «recordatorio" telefónico, como se expondrá en el apartado 1.A. 
tionario, el uso de incentivos y la insistencia en el anonimato de los cuestionarios produce incrementos en la colaboración de los entrevistados independientemente del método de recogida de datos. Así, y aunque los factores expuestos en esta primera parte afectan a todas las formas de recogida de datos, por limitaciones de espacio nos centraremos en las investigaciones postales, dejando para otro trabajo el análisis de la influencia de cada uno de estos elementos en la tasa y en la calidad de las respuestas obtenidas.

\section{A) Prenotificación}

El primer elemento que se encuentra el entrevistado (que todavía desconoce que lo es) es una comunicación notificándole que se está realizando una investigación sobre un tema determinado en la que se solicita su cooperación, al tiempo que se le informa el procedimiento de cómo ha sido incluido en la misma. La carta termina indicando que en unos días recibirá un cuestionario sobre el tema.

Podríamos plantear como hipótesis que la prenotificación incrementará la tasa de respuesta puesto que comunica a las personas que se está realizando una investigación sobre un determinado tema, al tiempo que establece una legitimación de la misma (Fox, Crask y Kim, 1988). Sin embargo, el análisis de las distintas investigaciones realizadas sobre este aspecto revela que la influencia de la prenotificación en la tasa de respuesta es distinta según el medio utilizado, postal o telefónico:

a) Hay un relativo acuerdo sobre la eficacia de la prenotificación telefónica (Jobber, Allen y Oakland, 1985; Wynn y McDaniel, 1985), pese a que otros trabajos más antiguos (Dillman y Frey, 1974) señalan que la prenotificación telefónica no aumenta significativamente la tasa de respuesta, aunque sí incrementa la rapidez en la contestación y reenvío de los cuestionarios.

b) El acuerdo sobre la incidencia de la prenotificación postal es mayor. En uno de los últimos trabajos sobre el tema realizado por Dillman, Clark y Sinclair (1995) se analiza la influencia de diferentes elementos en el aumento de la tasa de respuesta del Censo de 1992, consiguiendo con la prenotificación postal un incremento medio en la tasa de respuesta del 6,4 por 100 , frente al 2,6 por 100 conseguido tras enviar una tarjeta de recuerdo. Por otro lado, Fox, Crask y Kim (1988) afirman que la prenotificación postal puede llegar a producir incrementos del 47,4 por 100 en la tasa de respuesta.

En esta línea, y tras comparar la incidencia de ambas prenotificaciones en una muestra de 495 hogares, Faria (1990) concluye que el precontacto por carta es más efectivo que el telefónico y que el no precontacto: tasas de res- 
puesta $47,9,42,3$ y 33,7 por 100 , respectivamente. Este autor señala que no hay diferencia significativa entre la velocidad de respuesta, aunque la telefónica produce una respuesta algo más rápida. En lo referente a la calidad de la respuesta $^{5}$ obtenida tras la prenotificación, este autor no encuentra relación significativa entre la prenotificación y el número de ítems que se quedan sin contestar, aunque el precontacto telefónico es el peor (índice medio de ítems no contestados 0,275$)$, seguido del no precontacto $(0,193)$ y del postal $(0,141)$. Conclusiones similares han sido expuestas por Jobber, Allen y Oakland (1985) y Wynn y McDaniel (1985), negando así la relación entre prenotificación y aumento de la calidad en la respuesta.

No sólo es importante el medio por el que se realiza la prenotificación, sino que los efectos provocados por la misma varían según los organismos que la remiten, como ponen de relieve Brunner y Carrol (1988) cuando señalan que si es enviada por una universidad la tasa de respuesta aumenta del 52,3 al 72,5 por 100; mientras que cuando el remitente es una institución privada se produce una disminución de la respuesta del 53,9 al 47,7 por 100 .

Concluiremos este apartado señalando que el aumento en la tasa de respuesta producido por la prenotificación aconseja la utilización de una prenotificación postal, mejor que la telefónica, aunque debemos ser prudentes en la utilización de la misma puesto que esta efectividad puede variar según la institución que la envíe. No obstante, y pese a la influencia positiva de la carta de prenotificación en la mayoría de las situaciones, aconsejamos prudencia en el uso de las prenotificaciones cuando se realicen investigaciones en determinados colectivos (por ejemplo, grupos de profesionales, etc.), puesto que el efecto puede ser contraproducente al actuar la prenotificación como un "aviso" de algo que va a llegar y que podrá suponer un gran costo de tiempo y un escaso beneficio (Jobber, 1986).

\section{B) Aumento del número de contactos}

Existe un acuerdo en que el número de contactos que se realizan con el entrevistado eleva tremendamente la cooperación en las investigaciones mediante encuestas independientemente del medio utilizado (Dillman y Frey, 1974; Heberlein y Baumgartner, 1978; Dillman, 1991; Groves, Cialdini y Couper, 1992). Pese a que anteriormente ya se ha explicado la importancia de enviar una notificación previa al envío del cuestionario animando a cooperar (lo cual supone un primer contacto con el entrevistado antes aún de la llegada del cuestionario), en este apartado nos referiremos a la influencia de los contactos posteriores al primer envío del cuestionario. La costumbre de enviar un cuestionario y esperar a los que quieran contestar es una práctica totalmente desechada hoy en día. Más bien se trata de lo contrario: volver a comunicar

5 Definida según el número de ítems que se quedan sin contestar (Faria, 1990). 
con el entrevistado para dar más énfasis a su respuesta, creando la sensación de que su respuesta es tremendamente importante e insustituible. El hecho que algunos autores utilicen en algunos de los envíos posteriores (fundamentalmente en el último) algún tipo de correo «especial», como el correo certificado o el urgente, no hace más que enfatizar esa idea.

Uno de los primeros trabajos sobre el tema demostró que el uso de una estrategia mixta de recuerdo combinando correo certificado con llamadas telefónicas producía una tasa de respuesta del 83 por 100 , mientras que la utilización individual del correo certificado consigue una tasa de respuesta del 74 por 100 , y el teléfono (sin correo) de un 64 por 100 (Eckland, 1965) ${ }^{6}$. Más importante es el trabajo realizado por Heberlein y Baumgartner (1978), en el que, tras analizar los resultados de 98 investigaciones, concluyen afirmando que el número de contactos tiene una correlación del 0,63 con la tasa de respuesta. Ninguna otra variable de las 55 que recogen en su estudio muestra una correlación tan alta.

Dillman, en el Total Design Method (1978), propone un curioso sistema de implementación basado en el envío de una tarjeta postal una semana después del cuestionario a todos los entrevistados agradeciéndoles su cooperación y recordando la importancia de esta cooperación a los que todavía no lo han complementado. La mayoría de los autores están de acuerdo en mandar este primer recordatorio siete o diez días después del envío del cuestionario, aunque otros señalan que el mejor momento para enviarlo es después de haber recibido algunos cuestionarios (Paliwoda, 1981). En cuanto al efecto de este primer recordatorio en la tasa de respuesta, se estima entre un 17-22 por 100 (Brennan y Hoek, 1992). En el estudio realizado en el Departamento de Sociología de la Universidad Pública de Navarra se consiguió una tasa de respuesta del 29,5 por 100 tras el primer recordatorio.

Bien es verdad que si se piensa enviar más de un recordatorio es aconsejable incluir una copia del cuestionario por si se ha perdido el anterior, lo han tirado a la basura, etc. Dillman (1978) propone enviar tres semanas más tarde del primer envío - y únicamente a los que no han contestado- un nuevo cuestionario con una carta recordándoles que el cuestionario todavía no ha sido recibido. Brennan y Hoek (1992) señalan que el segundo recordatorio produce un incremento en la respuesta que oscila entre un 18-26 por 100. Este segundo recordatorio generó un 21,9 por 100 de respuestas en el estudio realizado en Navarra.

Cuatro semanas más tarde (siete semanas desde que se ha enviado el primer cuestionario), Dillman aconseja volver a enviar, esta vez por correo certificado para enfatizar más la importancia de la investigación, otro cuestionario con una carta en la que se insiste en la importancia de la colaboración del entrevistado en esta investigación, al tiempo que se expone de nuevo la enorme

"No olvidemos el año de realización del estudio (1964) y la tasa de posesión de teléfono en esos años: en 1960, el 80 por 100 de los hogares americanos tenía teléfono (Frey, 1989). 
importancia de su opinión. Dillman señala, recogiendo aspectos de la teoría del intercambio social, que el elevado coste económico del correo certificado genera un mayor gasto del investigador para incrementar la cooperación en la respuesta. Este incremento del coste económico, unido a la escasa utilización del correo certificado, aumenta la importancia de la investigación y crea en el entrevistado un sentimiento de autovaloración de su opinión. Por último, numerosos estudios han desaconsejado aumentar el número de envíos puesto que el incremento marginal en la respuesta del cuarto o quinto recuerdo es muy bajo (Dillman, 1978; Heberlein y Baumgartner, 1978).

No hay ninguna duda de la gran incidencia de este factor en el aumento de la respuesta, aunque todo este proceso supone un notable encarecimiento del trabajo de campo (hay personas que llegan a recibir cinco comunicaciones) y, evidentemente, la necesidad de dedicar más tiempo en la recogida de datos; llegando a necesitar dos meses con el modelo de Dillman. Respecto a este modelo hay que tener en cuenta que Dillman elaboró estos «tiempos de espera” pensando en la realidad de su país (Estados Unidos), en el cual las distancias geográficas son enormes. Si tenemos en cuenta las dimensiones de España y el hecho que el 79 por 100 de las cartas urbanas y un 67 por 100 de las provinciales llegan al día siguiente (Bosh y Lorente, 1993), consideramos que podrían acortarse los «tiempos de espera» entre los envíos, fundamentalmente entre los últimos. Está claro que en caso que pensemos modificar estos «tiempos de espera" habrá que tener en cuenta el ámbito geográfico de la muestra (nuestra ciudad, provincia o todo el país) y el tiempo que necesita el sobre de respuesta para regresar hasta el investigador.

\section{C) Incentivos}

El incremento que experimenta la respuesta tras entregar un regalo o dinero en metálico es uno de los factores en los cuales el acuerdo es mayor, aunque ha sido también uno de los factores que más tarde ha comenzado a ser investigado, como lo prueba el hecho que la mayoría de las referencias localizadas son posteriores a la década de los setenta (Church, 1993). Los incentivos utilizados para aumentar la cooperación de los entrevistados pueden clasificarse, a grandes rasgos, en dos grandes grupos según el momento de la entrega (antes de responder el cuestionario - prepago - y una vez enviado el cuestionario - promesa-) y el tipo del incentivo: monetarios y no monetarios (bolígrafos, lotería, donativos a instituciones benéficas, ofrecer un resumen de los resultados $^{7}$, café, libros, despertadores, pelotas de golf, sellos, pins, etc.).

El envío de un incentivo con el primer cuestionario - antes que el entrevistado decida si lo va a contestar- está fundamentado en la existencia de una

Este aspecto se analizará con más detalle en el apartado 2.C): la carta de presentación que acompaña al cuestionario. 
norma de reciprocidad según la cual tras el inicio de una relación social las personas se sienten obligadas a dar "algo" cuando reciben un premio. Así, el efecto combinado de la reciprocidad y el principio de la justicia redistributiva incidirá tremendamente en que el prepago incremente las tasas de respuesta. Furse y Stewart (1984) han realizado aplicaciones de la teoría de la disonancia cognoscitiva al estudio de la respuesta en las encuestas postales: desde su punto de vista, las tasas de respuesta pueden incrementarse creando una sensación de disonancia entre los entrevistados (el recibir un incentivo crearía esta situación), disonancia que puede ser resuelta al contestar el cuestionario y enviarlo al investigador.

Un metaanálisis realizado por Church (1993) en el que recoge 74 investigaciones realizadas en los últimos cincuenta ańos muestra que tanto los incentivos monetarios como los no monetarios aumentan significativamente la tasa de respuesta, aunque los incluidos con el primer cuestionario (prepago) producen el mayor incremento de la respuesta. Una de las principales conclusiones del estudio es que influye más la forma de recibir el incentivo (antes o después de contestar) que el tipo de incentivo recibido, produciendo incrementos de respuesta superiores al 15 por 100 (Linsky, 1975; Hopkins y Gullickson, 1992). La utilización de un incentivo monetario en el primer envío genera un incremento medio en la respuesta del 65 por 100 (Church, 1993). En cuanto a la influencia de la magnitud del incentivo en la tasa de respuesta debe quedar claro que el incentivo supone un "premio" para el entrevistado; es algo simbólico (Linsky, 1975; Hopkins y Gullickson, 1992) y en ningún momento adopta el papel de un «salario" o pago por un servicio o por el tiempo empleado.

Como conclusión de la influencia de este factor, señalar la gran importancia de los incentivos incluidos con el primer cuestionario y el hecho que éstos sean monetarios, aunque éste es un aspecto que influye en menor medida. Planteábamos al principio de este trabajo que una de las ventajas de las encuestas postales es su reducido costo económico. Ahora bien, ¿se mantiene éste bajo tras la utilización de incentivos económicos? Debe quedar claro que el incentivo es una gratificación por la dedicación del entrevistado, que en ningún momento adopta el papel de un "salario" o pago por un servicio o por el tiempo empleado. Una gran cantidad de trabajos demuestran cómo el incremento que se produce en la tasa de respuesta es mucho menor que el coste económico que supone la utilización de incentivos monetarios ${ }^{8}$. Así, y aunque uno de los problemas del incentivo es que aumenta el costo de la investigación, este encarecimiento en ningún momento llega a igualar al coste de la encuesta personal. Para finalizar, si tenemos en cuenta que el incentivo puede aumentar

${ }^{*}$ Entre éstos destacamos el trabajo de Hopkins y Gullickson (1992), en el que encuentran que no hay una relación lineal entre aumento del incentivo y aumento de la tasa de respuesta: los incentivos menores de medio dólar producen un aumento en la respuesta del 13,3 por 100; entre medio dólar y un dólar el aumento es del 19,4 por 100; incentivos entre uno y dos dólares mejoran la respuesta en un 22,1 por 100 , y cuando se envían más de dos dólares el aumento es del 29,4 por 100; todo ello medido en dólares de 1990 . 
la respuesta hasta en un 65 por 100, hemos expuesto uno de los factores con más influencia en la tasa de respuesta.

\section{D) Anonimato}

El principal problema que se plantea con este aspecto es que el entrevistado pueda variar sus respuestas por el temor de los efectos de sus respuestas. Así, la necesidad de incluir un apartado sobre "anonimato" tiene su origen no sólo en el hecho que los cuestionarios suelan tener nombres y apellidos, sino fundamentalmente porque cada cuestionario viene identificado con un número para que el investigador conozca los que han contestado y los que no, y así poder tenerlos en cuenta para enviar posteriormente un nuevo cuestionario, otra carta de recuerdo, una gratificación, etc. Aunque existen diversas estrategias para colocar este número en lugares poco perceptibles para el entrevistado, por ética profesional consideramos que no se debe ocultar y, de hecho, numerosos autores han aconsejado colocarlo en la parte exterior derecha de la primera página del cuestionario con el fin que sea visto claramente. Normalmente, en la carta de presentación se dedica un espacio para insistir en el anonimato del cuestionario, explicando la razón y el uso de este número identificativo.

Los resultados de la literatura existente muestran divergencias en los hallazgos realizados: investigaciones realizadas por Scott (1961) y Singer (1978) señalan que el anonimato incrementa la tasa de respuesta. No obstante, otros trabajos indican que existe una escasa influencia del anonimato en las respuestas (Janssens y Pessemier, 1980; McDaniel y Rao, 1981; Scott, 1993). Es la variabilidad en los objetos de estudio, y fundamentalmente en la selección de la población entrevistada, la causante de estos resultados tan dispares: tras seleccionar los estudios que tienen como objeto de estudio colectivos no especializados (Jones, 1979; Scott, 1961; Singer, 1978), la mayoría muestra una influencia del anonimato en la tasa de respuesta (Singer, 1978; Scott, 1961).

Además de la influencia en la tasa de respuesta, el anonimato puede producir cambios en la calidad de la misma, como señalan McDaniel y Rao (1981) cuando concluyen que la información suministrada por los entrevistados anónimos será peor que la obtenida por los no anónimos: los entrevistados anónimos dan menos información exacta que los entrevistados identificados. Downs y Ker (1986) utilizan una muestra de la población general para resolver las siguientes hipótesis: a) el anonimato no influye en el sesgo de la respuesta; b) el anonimato no influye en la no respuesta de algunas preguntas, y c) la calidad de la respuesta ${ }^{9}$ será menor en los cuestionarios "anónimos». Sus conclusiones permiten aceptar la primera hipótesis, aunque hay algunas diferencias sociodemográficas - que no llegan a ser significativas- entre los que escriben

? Calidad de la respuesta entendida como una lectura adecuada de las instrucciones por parte de los entrevistados. 
el remite en el cuestionario y los que no lo hacen (anónimos). Respecto a la segunda hipótesis, apenas hay alguna diferencia en la respuesta parcial entre ambos colectivos. En cuanto a la calidad en la respuesta, los anónimos tienden a dar menos información que los que escriben su remite en el cuestionario.

Damos por terminado el análisis del anonimato señalando que incrementa la tasa de respuesta, aunque los cuestionarios anónimos suelen estar peor cumplimentados que el resto. No obstante, la ventaja es que las personas que contestan cuestionarios anónimos se muestran más críticas a la hora de exponer sus opiniones, animando con ello a utilizar, siempre que sea posible, cuestionarios anónimos. Cuando por alguna razón sea imposible enviar cuestionarios anónimos será conveniente insistir en la confidencialidad en las respuestas, fundamentalmente cuando el cuestionario pregunte sobre información «sensible».

Planteábamos al principio de este trabajo que utilizando una serie de factores podemos lograr que la calidad de los datos recogidos mediante encuestas postales sea similar a otros métodos de recogida de datos. Como se ha puesto de manifiesto en esta primera parte, el uso de estas cuatro estrategias incrementará notablemente la tasa y la calidad de la respuesta, permitiendo verificar parcialmente la hipótesis planteada. En la exposición se ha analizado la importancia de cada una por separado; qué duda cabe que la utilización conjunta tendrá una incidencia notablemente superior. Debemos recordar que cada una de éstas puede ser utilizada con cualquier método de recogida de datos, aunque existen pocas referencias bibliográficas que den cuenta de la utilización de éstas con otros métodos de recogida de datos. A excepción de la insistencia en el anonimato y la utilización esporádica de la prenotificación, estos factores son muy poco utilizados en las encuestas personales y telefónicas, con lo cual nos da pistas sobre las razones por las cuales se está reduciendo la tasa de cooperación en los últimos años.

A partir de este momento se expondrán factores propios de las encuestas postales, aunque algunos de ellos (como el diseño del cuestionario) pueden afectar también a las encuestas personales. En el próximo apartado se analizará la «imagen» del investigador que reciben los entrevistados (a través de los medios que tiene para ello) y cómo influye cada uno de éstos en la calidad de los datos recogidos.

\section{CARACTERISTICAS PECULIARES DEL MEDIO \\ DE ADMINISTRACION POSTAL. \\ LA «TOMA DE CONTACTO» MEDIANTE EL CORREO}

La peculiaridad de las investigaciones postales es que no hay contacto personal entre el investigador y los entrevistados a través de los entrevistadores. El efecto - positivo o negativo- de los entrevistadores queda fuera de análisis en 
las encuestas postales ${ }^{10}$. Así, aspectos como el sobre y el tipo de correo utilizado, la carta de presentación de la investigación, la personificación de la correspondencia y el sobre de reenvío se convierten en la imagen que el investigador transmite de su proyecto. Esta imagen influirá en la cooperación de los entrevistados, como vemos a continuación.

\section{A) El sobre y tipo de correo utilizado}

Cuando una persona llega a su casa y recoge el correo se encuentra, entre una gran variedad de folletos publicitarios y una multitud de mensajes que buscan aumentar las ventas de determinados productos, una carta personal ${ }^{11}$ que no esperaba recibir. Tras el análisis del sobre, el remitente (ver apartado 4) y algunos otros factores, puede decidir abrirla o tirarla a la basura que - en muchos edificios - se encuentra junto a los buzones.

Entre los factores del TDM, Dillman (1978) señala la gran importancia de utilizar correo de "primera clase" para el primer envío de los cuestionarios, eliminando el llamado "correo masivo" sin sello, puesto que da la sensación de masificación en un medio en el que la personalización es muy importante, como se expondrá en el siguiente apartado. Algunos autores han utilizado correo certificado o urgente para el primer contacto, aunque el incremento en la tasa de respuesta que se consigue con esta medida no es proporcional al aumento del coste económico del mismo. Es por ello por lo que la mayoría de los autores aconsejan utilizar este tipo de correo en las últimas fases del estudio, para así incidir con este medio en la importancia de la respuesta del entrevistado (Heberlein y Baumgartner, 1978).

En cuanto al tipo de sobre utilizado, Dillman aconseja un tamaño estándar para que pueda ser enviado con una tasa de franqueo normal y así ser más económico que sobres grandes, además de dar la sensación que el cuestionario incluido en un sobre pequeño será breve y podrá ser rápidamente contestado. Esto es muy importante cuando se ha enviado una prenotificación y se envían las cartas de recuerdo, puesto que el entrevistado puede saber que el contenido de esta carta remitida por ese organismo es un cuestionario.

Apenas se ha investigado sobre la influencia del tipo de sobre en la respuesta, el color del mismo, las dimensiones y otros factores como los distintos "mensajes» que pueden ser añadidos al sobre: respuesta inmediata, muy importante, etc. Aunque Dillman (1978) aconseja utilizar sobres de distintos tamaños y colores, las características de la correspondencia que recibimos habitualmente, unido al hecho que las empresas de publicidad y mailing cada vez dis-

10 Burchell atribuye a la pericia de los entrevistadores los aumentos de calidad de determinadas partes del cuestionario (1992: 241-243).

"Puede ser también una carta no personal (dirigida a los residentes del $3 .^{\circ} \mathrm{A}$ ), como se expone en el próximo apartado. 
ponen de más medios para hacernos llegar sobres de diferentes tamaños y con distintos colores para llamar más la atención, nos lleva a la conclusión que lo mejor es un sobre blanco de buena calidad a fin de diferenciarlo del resto de papeles que el posible destinatario se puede encontrar en el buzón. Por otra parte, y respecto a los posibles "mensajes exteriores del mismo" (muy importante, abrirlo inmediatamente, respuesta inmediata, etc.), creemos que es mejor la no utilización de éstos a fin de diferenciarlo de los mensajes publicitarios, y porque dan la sensación de una premura que no es tal. Desde nuestro punto de vista, estos mensajes no aumentan la sensación de importancia de la investigación, aunque insistimos en que no se han realizado investigaciones al respecto, con lo cual animamos a las personas interesadas en estos temas a profundizar en este aspecto.

Relacionado con lo anterior, es muy importante que el sobre contenga el nombre y la dirección del entrevistado, aunque si no se conoce podría ponerse «a la familia del número 54 de la Calle Mayor», por ejemplo. NUNCA se debe enviar el sobre sin un destinatario. Tanto la carta de presentación como el propio sobre deben tener un destinatario, con nombre o sin él. Como todos conocemos, la probabilidad de que una carta dejada en nuestro buzón sin ninguna referencia hacia nosotros sea leída y tomada en serio es muy baja.

El cálculo de la tasa de respuesta nos obliga a conocer con la máxima exactitud posible el número de personas que han recibido el cuestionario, eliminados aquellos con dirección incorrecta, incompleta, desconocidos, etc. Es por ello por lo que se aconseja añadir en la parte de atrás del cuestionario un recuadro en el que el cartero pueda indicar la causa de la devolución, si ésta se produce $^{12}$. Comparado con otros países, éste no es un gran problema en nuestro país puesto que la tasa de cambio de residencia es muy baja, sobre todo cuando se compara con otros países como Estados Unidos, que es donde más se ha utilizado esta forma de recogida de datos.

\section{B) Personalización de la correspondencia}

Personificar la correspondencia da la sensación que la carta ha sido enviada individualmente a cada uno de los destinatarios, generando una percepción de que su opinión es única y tiene una importancia singular, eliminando así la impresión de masificación que produce una carta enviada «a todo el mundo». Son varios los elementos que intervienen en la personificación: en primer lugar, la escritura del nombre y apellido del destinatario en el sobre donde recibe la correspondencia (escritura, no el pegado de una etiqueta), así como el «tipo» de escritura realizada: a máquina o a mano ${ }^{13}$. También en la carta de

12 Sr. Cartero: Por favor, indique con una X la causa de la devolución: Desconocido, Rehusado, Ausente, Dirección incompleta.

${ }_{13}$ Nederhof (1983) ha demostrado que personificar el exterior del sobre por medio de direcciones escritas a mano aumenta notablemente la respuesta. 
presentación de la investigación hay diversos elementos que indican el grado de personalización de la correspondencia: la dirección del destinatario, una primera referencia a una persona concreta (Estimado Sr. Pérez), la despedida, el agradecimiento personal y la firma de la misma (firma real con bolígrafo de otro color, firma "impresa" con otro color, firma del mismo color que el resto de la carta).

Podría considerarse la personalización como un efecto opuesto al anonimato puesto que el hecho de recoger un sobre sin un destinatario claro (p. ej., "los residentes del $3 .^{\circ} \mathrm{A}$ ) u otro con nombre y apellidos, el último puede generar en los entrevistados una pérdida de anonimato. Los entrevistados pueden llegar a desconfiar de las promesas de confidencialidad de un investigador que conoce su nombre. $\mathrm{O}$, por lo menos, tendrán más dudas que los que han recibido la carta con la indicación "para los residentes del 3. A".

La mayoría de las investigaciones realizadas señalan una gran relación entre la prenotificación y el aumento de la respuesta ${ }^{14}$ con incrementos de respuesta en torno al 8 por 100 (Linsky, 1965; Carpenter, 1974), llegando en determinados momentos al 25 por 100 (Roeher, 1963). Otros autores (Simon, 1967; Watson, 1965; Andreason, 1970) han señalado escasos aumentos e incluso descensos en las tasas de respuesta, llegando a afirmar en determinados momentos que la personalización puede ser contraproducente (Andreason, 1970). Una explicación de esta variabilidad en los resultados se logra tras analizar las peculiaridades muestrales de estos trabajos (personas que han recibido premios de lotería y trabajadores de una compañía) y la gran cantidad de preguntas sensibles incluidas en cada uno de éstos. La necesidad de anonimato requerida por estas temáticas contrasta fuertemente con el acierto de enviar cartas personalizadas.

Uno de los últimos trabajos realizados sobre el tema, que es además una de las escasas investigaciones realizadas fuera del Reino Unido o Estados Unidos, trata de analizar la influencia de la personificación en la tasa y calidad de la respuesta de una muestra de la población general en Holanda (DeLeeuw y Hox, 1988): la no personificación reduce la tasa de respuesta un 10 por 100 , aunque por otro lado reduce también la influencia de la deseabilidad social en las respuestas ${ }^{15}$. En cuanto al número de preguntas no respondidas, no hay diferencias significativas entre los que reciben la prenotificación y los que no lo hacen.

Pese a la incidencia positiva de la personificación en la tasa de respuesta, concluiremos este apartado con un consejo impartido por Simon (1967) cuando dice que los efectos de la personificación dependen del tipo de población analizada y los objetivos del cuestionario, aspecto que ya se ha puesto de relie-

'Longworth (1953); Scott (1961); Linsky (1965); Simon (1967); Carpenter (1974); Dillman y Frey (1974); Cox, Anderson y Fulcher (1974); DeLeeuw y Hox (1988).

Adoptando la definición de "Deseabilidad Social" de DeMaio (1984): "Una pregunta puede provocar un efecto de "deseabilidad social" si sus respuestas están altamente influenciadas por expectativas sociales." 
ve en la exposición del mismo. Por ello aconsejamos prudencia en la utilización de la personalización cuando se realizan preguntas sensibles y cuando el objeto de investigación esté físicamente cercano, como es el caso (por ejemplo) de una valoración de las condiciones de trabajo por parte de los trabajadores, satisfacción con el ambiente de la empresa, etc.

\section{C) La carta de presentación}

Otro de los elementos que implementan la eficiencia en las encuestas postales es la carta de presentación de la investigación. Esta carta de presentación no sólo informa sobre el tema, sino que solicita la cooperación por parte del entrevistado, además de explicar otros elementos de indudable importancia para él. La carta comienza con un encabezado de la organización responsable de la investigación, la fecha (debe ser lo más actual posible) y el nombre y los apellidos (recordar el apartado personalización) del destinatario. Estas cartas no tienen por qué seguir el orden que exponemos aquí, aunque sí es importante no olvidar ninguna de estas partes.

Veamos: en el primer párrafo del texto se explican los objetivos del estudio, tratando de convencer al entrevistado de la utilidad e importancia de este estudio en su ámbito personal, laboral, social, etc. Posteriormente se explica cómo ha sido seleccionada esa persona y se vuelve a incidir en la importancia de sus respuestas. En el siguiente párrafo se le explica la razón por la que el cuestionario está numerado - ver anonimato y apartado 1.C)_-, insistiendo en que el cuestionario es anónimo y que sus respuestas serán tratadas confidencialmente. Tras esto se suele decir al entrevistado que - si lo desea- puede recibir un resumen de los resultados cuando la investigación esté finalizada (incentivo no monetario $)^{16}$, al tiempo que se vuelve a insistir en la importancia del mismo. Antes de la despedida, algunas cartas suelen incluir una frase en la que ofrecen un teléfono (generalmente gratuito) al que llamar si hay alguna duda. Por último, se le agradece al entrevistado su colaboración y se firma al final de la carta. Bajo la firma debe estar escrito el nombre del director del trabajo y su cargo ${ }^{17}$.

Éste es el aspecto al que se ha dedicado un menor número de estudios, aunque no por ello dejamos de encontrarnos con resultados contradictorios.

16 Dillman (1978) señala la importancia de ofrecer este incentivo no monetario en un tema en el que los entrevistados pueden estar interesados. Desde su punto de vista, el hecho que más de la mitad de los entrevistados (entre un 50 y un 66 por 100) soliciten una copia de los resultados significa que muchos entrevistados valoran el hecho de poder ver los resultados del estudio, constituyendo así un excelente estímulo para responder.

17 Algunos expertos aconsejan insistir en la carta de presentación que el cuestionario debe ser respondido y enviado antes de una determinada fecha, puesto que ciertos experimentos han señalado que este hecho incrementa la tasa de respuesta (Futrell y Hise, 1982; Henley, 1976; Vocino, 1977), si bien investigaciones más recientes han señalado que si se pide al entrevistado que conteste con urgencia el nivel de cooperación baja (Smith, 1995; Steeh, 1981). 
Pese a que las escasas investigaciones realizadas indican que la apariencia de la carta de presentación influye en el incremento de la respuesta (Houston y Nevin, 1977; Jones y Linda, 1978; Robertson y Bellenger, 1978), los estudios de Champion y Sear (1969) y (Linsky, 1965 y 1975) proporcionan ligeras evidencias para manifestar lo contrario.

Algunos trabajos señalan que la tasa de respuesta está influenciada por el mensaje de las cartas de presentación (Hornik, 1981), mientras que otros que han analizado el efecto de establecer una "comunicación" a través de la carta de presentación (proporcionando una dirección o un teléfono gratuito para las dudas) señalan que este hecho tiene una gran importancia en la respuesta (Powwers y Alderman, 1982). Desde otra perspectiva, Robin y Walters (1976) afirman que cuando la carta está acompañada de un incentivo las variaciones en el mensaje de la misma no producen cambios en la tasa de respuesta. Scott (1961) asegura que imprimir la carta de presentación en la misma hoja que el cuestionario produce un significativo aumento en la tasa de respuesta (95,8 por 100 , frente a 93,6 por 100 ). Otro de los aspectos que tiene gran importancia dentro de la carta de presentación es el color de la firma del que la envía (Vocino, 1977), razón por la que la mayoría de los autores aconsejan firmar cada una de las cartas.

Pese a la influencia de este factor, es sin duda uno de los que necesita más investigación, puesto que la escasa acumulación de resultados nos impide evaluar la importancia de determinados factores. El problema no es que los hallazgos expuestos por las investigaciones realizadas no estén suficientemente sustentados, sino que - como se ha señalado anteriormente- en numerosas ocasiones se extraen conclusiones de colectivos específicos que no permiten generalizar sus aportaciones a muestras de población general. Sin duda, esta falta de investigaciones sobre este tema es debida a que - hoy en día- nadie envía una encuesta postal sin una carta explicativa de la misma.

\section{D) Reenvío}

Aunque hay un gran número de trabajos referidos a la importancia del tipo de correo utilizado (tarifa postal) en la probabilidad que el destinatario reenvíe el cuestionario o no, el acuerdo en la influencia de este factor es escaso: diversas investigaciones realizadas en los años cincuenta afirmaban que el correo ordinario tiene mayor tasa de respuesta que el franqueo en destino (Longworth, 1953; Gullahorn y Gullahorn, 1959). En el primer quinquenio de los años ochenta otros estudios han mostrado resultados opuestos: McCrohan y Lowe (1981) sostienen que no existe diferencia entre las tasas de respuesta cuando se utilizan distintos tipos de franqueo, y Labrecque (1978) afirma que la utilización de sellos conmemorativos no tiene impacto alguno en la tasa de respuesta, e incluso algunos llegan a señalar que tiene un impacto negativo (Vocino, 1977).

La enorme confusión en las conclusiones proporcionadas por estos trabajos 
queda despejada al analizar uno de los últimos artículos publicados sobre el tema, en el que se exponen los resultados obtenidos por 34 investigaciones realizadas entre 1951 y 1986 respecto a la influencia del tipo de sello en el sobre de respuesta (Armstrong y Lusk, 1987). Estos autores comparan las respuestas obtenidas en cada una de estas 34 investigaciones postales según el franqueo del sobre de respuesta: sobre de respuesta sin sello, "a franquear en destino", con sello "conmemorativo" de algún acontecimiento y con sellos normales. Los resultados muestran, como era de esperar, que el sobre sin sello es el que obtiene una menor respuesta. El sobre "franqueado en destino" consigue una respuesta mayor, pero menor que el resto de correo en un 9,2 por 100. Tras comparar los distintos tipos de franqueo disponible en Estados Unidos con los "sellos normales", estos últimos muestran su superioridad al recibir un 3 por 100 más de respuestas. Por último, los sellos conmemorativos aumentan ligeramente la tasa de respuesta: un 1,6 por 100 superior a los sellos normales. Similares conclusiones fueron expuestas por Fox, Crask y Kim (1988), llegando a afirmar que la utilización de sellos —en vez del "franqueado en destino"puede incrementar la tasa de respuesta hasta en un 32 por 100.

Pese al aumento del sello frente al "franqueado en destino", debemos tener en cuenta que el sello encarece tremendamente el coste de los trabajos de campo, puesto que si tenemos en cuenta que la tasa de respuesta de la primera oleada puede llegar (en el mejor de los casos) al 48,3 por 100 (Nederhof, 1988), se "pierden" unos 620 sellos en una muestra de 1.200. En la segunda oleada, con una tasa acumulada de respuesta del 65,6 por 100 , quedan sin utilizar 413 sellos, y en la tercera (tasa final de respuesta del 69,5 por 100) no llegan a utilizarse 366 sellos. En total, 1.399 sellos que se quedan sin utilizar, que al coste actual son 48.965 pts. Así, la comparación con el coste del franqueado en destino ( 35 pts. por cada sobre más las 8.015 que cuesta el sistema de "franqueo en destino") nos permite un notable ahorro económico. Consideramos que en este factor es el propio investigador el que debe elegir entre utilizar un sobre con sello y conseguir una tasa de respuesta superior, o reducir los gastos utilizando un sobre a franquear en destino

Recordando que nuestro objetivo es conocer los factores que mejoran la respuesta de las encuestas postales, el análisis de las aportaciones de esta segunda parte del trabajo indica que la influencia de estos factores es menor, aunque en todo momento se trata de factores que inciden positivamente en la respuesta. Respecto al sobre y tipo de correo, la gran cantidad de publicidad que recibimos en nuestros hogares nos hace preguntarnos hasta qué punto puede ser abierto y leído un sobre con una encuesta postal, aunque el enviarlo dentro de un sobre blanco con un determinado remitente contribuye significativamente en un incremento de la respuesta, como se ha demostrado en el apartado 1.d). La personificación de la correspondencia, por otro lado, genera en el entrevistado una sensación de que su opinión es importante. Este aspecto, unido al hecho de recibir más de una comunicación e incluso un correo certificado, produce una mayor autovaloración del entrevistado y la sensación que esta 
investigación es muy importante. Pese a la escasa investigación realizada sobre la carta de presentación, consideramos que es un aspecto fundamental puesto que sirve para explicar la finalidad de la investigación y aclarar todas las dudas que pueden surgir. Como se ha señalado, la causa de esta falta de investigaciones sobre el tema está en que nadie envía un cuestionario sin una carta de presentación. Por último, el sello y sobre de respuesta tienen una gran influencia, consiguiendo el sello normal una tasa de respuesta superior que el resto del franqueo (en las investigaciones realizadas en Estados Unidos).

\section{INCIDENCIA DEL CUESTIONARIO}

En este apartado se expondrán diversos elementos relacionados con el cuestionario como son su longitud y el diseño del mismo: formato, diseño de portada y contraportada, tipo de impresión final y color del papel. Estos elementos adquieren suma importancia porque en ningún otro método de recogida de datos los entrevistados se encuentran físicamente con el cuestionario. Este hecho genera que los entrevistados se encuentren (o se «enfrenten") en primer lugar con un cuestionario; en un segundo momento concentrarán su atención en los aspectos visuales del mismo (quizás pueden comenzar a leerlo), y, por último, decidirán contestarlo o no ${ }^{18}$. Ésta es la razón por la que los aspectos visuales y la facilidad de las preguntas tienen una importancia capital en los cuestionarios postales (Jenkins y Dillman, 1996). El proceso de respuesta sigue una sucesión de pasos, mientras que en el cuestionario personal o telefónico no se produce esa evaluación. La decisión de aceptar (o rechazar) colaborar en la investigación es más inmediata, y la presión social (el no quedar mal) puede llevar a no interrumpir la secuencia del cuestionario aunque el entrevistado se encuentre incómodo en esa situación. Veamos la incidencia de distintos elementos del cuestionario en la tasa y calidad de la respuesta.

\section{A) Diseño del cuestionario: aspectos visuales}

La exposición sobre el diseño del cuestionario va a ser dividida en varias partes, comenzando con la influencia del tamaño del formato del cuestionario, para continuar con otros elementos como el diseño de la portada y contraportada, el tipo de impresión final, el color del cuestionario y la precodificación de las preguntas. Debemos señalar que no se han realizado muchas investigaciones para analizar la influencia de cada uno de estos factores, de modo que en ciertos momentos será una única referencia la que aconseje introducir o eliminar determinados aspectos. En este sentido, animamos a los interesados en

${ }^{18}$ Furse y Stewart (1984) han desarrollado en un excelente trabajo cómo afecta cada uno de estos elementos a la respuesta, exponiendo también las estrategias para solucionarlo. 
el tema a realizar nuevos trabajos a fin de conocer cómo afectan cada uno de estos factores a la tasa y calidad de respuesta.

No hay muchas investigaciones dedicadas a analizar el tamaño del formato del cuestionario en la respuesta del mismo. Así, y pese a que Dillman (1974) propone que el mejor tamaño para el cuestionario es de $15,5 \times 21 \mathrm{~cm}$., en una obra posterior (1991) considera que este aspecto del TDM ha sido analizado en escasas ocasiones y anima a realizar más estudios sobre el tema. Por otro lado, Erdos y Mogan (1983), en una obra clásica sobre el tema, proponen un cuestionario algo mayor $(18,5 \times 25,5 \mathrm{~cm}$. $)$.

Dentro de la escasa investigación realizada sobre el tema destacamos un trabajo realizado en el Reino Unido (Jansen, 1985) en el que se compara la tasa de respuesta producida por diversos tamaños de un cuestionario administrado a la población general: el tamaño propuesto por Dillman $(15,5 \times 21 \mathrm{~cm}$.), el propuesto por Erdos y Morgan $(18,5 \times 25,5 \mathrm{~cm}$.) y un tamaño intermedio $(17 \times 24,5)$. El autor considera que el formato $17 \times 24,5 \mathrm{~cm}$. es el mejor al conseguir diferencias de respuesta superiores en un 9 por 100 a los otros tamaños. Johnson y otros (1993) realizan una réplica de esta investigación en Estados Unidos comparando el cuestionario de Jansen con un formato $21,6 \times 27,9 \mathrm{~cm}$. (papel estándar USA) en una muestra de médicos de familia. En su experimento, el tamaño $21,6 \times 27,9 \mathrm{~cm}$. consigue una mayor tasa de respuesta y un menor número de preguntas sin contestar. El análisis de éstos y otros resultados le llevan a Dillman (1991) a afirmar que cuestionarios de reducido tamaño son respondidos mejor por los jóvenes, mientras que la población de más edad prefiere cuestionarios de mayor tamaño.

Debemos prestar una gran atención a la portada y contraportada del cuestionario puesto que es lo primero que observa el entrevistado y condiciona la conducta de éste. En el Total Desing Method, Dillman (1974, 1978 y 1983) propone una portada con el título de la investigación sobre un dibujo o una ilustración atractiva que tenga un significado neutro. Bajo este dibujo puede ponerse una explicación sobre la investigación, un mensaje que recuerde la importancia de las opiniones del entrevistado, etc., y al pie de página el nombre y dirección de la institución que lo promueve. Nunca deben realizarse preguntas en la primera página, dejando ésta para el título y la presentación de la investigación. Respecto a la contraportada, este autor aconseja dedicarla para invitar a los entrevistados a realizar sugerencias, preguntas, etc. Tampoco deben incluirse cuestiones en este espacio. Pese a que la influencia de estos aspectos no ha sido muy estudiada, Nederhof (1988) demuestra que el dibujo de la cubierta del cuestionario incrementa la tasa de respuesta al aumentar la importancia atribuida al cuestionario ${ }^{19}$. Así, y aunque los recordatorios también incrementan la importancia del cuestionario, la primera impresión transmitida por la portada es muy importante (Grembowski, 1985; Nederhof, 1988).

19 Nederhof (1988) compara un mismo diseño de portada con fondo blanco (impresión en negro) y fondo negro (impresión en blanco), consiguiendo el segundo una tasa de respuesta un 15 por 100 superior al primero. 
Respecto al tipo de impresión final, Jansen (1985) no encuentra grandes diferencias entre las tasas de respuesta a un cuestionario realizado con máquina de escribir y otro realizado por un impresor profesional con sombreados, negritas, recuadros para la respuesta, etc. No obstante, y debido a que este cambio en el diseño aumentó el tamaño del cuestionario de 12 a 15 páginas, no es posible analizar separadamente el efecto del tipo de impresión. Mucho más cuando sobrepasa el tamaño "crítico" (en torno a las 10-12 páginas) a partir del cual la influencia de la longitud adquiere más importancia, como se expondrá en el siguiente apartado.

\section{Relación entre tasa de respuesta y color del cuestionario}

Tipo de población

a) Becarios

b) Organización profesional

c) Estudiantes

d) Directores de empresas textiles

e) Revisión de 10 estudios
Color del cuestionario

Tasas de respuesta

\begin{tabular}{|c|c|}
\hline Verde y blanco & 51 y 49 por 100 , respectivamente \\
\hline Rosa y blanco & 24 y 20 por 100 , respectivamente \\
\hline Amarillo-blanco & 49 y 37 por 100 , respectivamente \\
\hline Azul y blanco & $\begin{array}{l}\text { El azul consigue más respuestas, } \\
\text { aunque no hay grandes diferencias }\end{array}$ \\
\hline Verde y blanco & $\begin{array}{l}\text { El verde consigue una tasa de res- } \\
\text { puesta superior en un } 5 \text { por } 100 \text {. }\end{array}$ \\
\hline
\end{tabular}

FUENTES: a) Gullahorn y Gullahorn (1963).

b) Matteson (1974).

c) Crittenden, Crittenden y Hawes (1985).

d) Jobber (1986).

e) Fox, Crask y Kim (1988).

Aunque el uso de páginas de colores puede recordar a los entrevistados el correo masivo o de baja calidad y ser contraproducente (Pressley y Tullar, 1977), una gran cantidad de estudios indican que el color del cuestionario influye en la tasa de respuesta, tal y como se muestra en la tabla adjunta. Existen varias interpretaciones sobre esta influencia; Jobber (1986) señala que la utilización de hojas de colores añade al cuestionario un cierto toque de conspicuidad cuando se coloca en una mesa entre un montón de papeles (blancos en su mayor parte), mientras que otras explicaciones están relacionadas con los efectos que cada uno de los colores utilizados tiene en la mente de los entrevistados, que no vamos a citar aquí por estar fuera de los objetivos de este trabajo.

Por último, en lo que respecta a la precodificación de las preguntas, el consejo es no precodificarlas puesto que pueden crear confusión en el entrevistado, aunque algunas investigaciones (Stevens, 1974) no han encontrado relación entre precodificación de las preguntas y aumentos en las tasas de respuesta. 


\section{B) Longitud del cuestionario}

Aunque existen algunos trabajos donde se señala que los cuestionarios cortos consiguen mayores tasas de respuesta (Cartwright y Ward, 1968; Hansen y Robinson, 1980; Powwers y Alderman, 1982), el análisis de la mayor parte de la literatura sobre el tema indica ausencia de relación entre la longitud del cuestionario y la tasa de respuesta ${ }^{20}$, excepto cuando éste es muy largo. Tras analizar 50 investigaciones, Dillman (1978) no encuentra diferencia en las tasas de respuesta entre los cuestionarios menores de 12 páginas o 125 ítems. A partir de este tamaño la tasa de respuesta disminuye al 65 por 100 , con lo cual aconseja un cuestionario con 10-12 páginas como el más apropiado.

En dos investigaciones sobre actitudes hacia el suicidio y separación matrimonial, Jansen (1985) encuentra que los cuestionarios cortos producen una mayor tasa de respuesta que los largos (superioridad entre un 8-10 por 100). El diseño de este trabajo, en el que se analiza conjuntamente la longitud del cuestionario, el tamaño y la calidad de impresión del mismo, nos lleva a adoptar un planteamiento crítico puesto que no existe certeza clara de la influencia exacta del tamaño del cuestionario. El diseño del trabajo no permite separar el efecto individual de cada elemento, con lo cual dichos hallazgos deben ser analizados con suma prudencia.

Investigaciones realizadas por Champion y Sear (1969) para conocer el impacto de la longitud del cuestionario y los aspectos visuales del mismo han restado importancia a la longitud del cuestionario. Estos autores, una vez decididas las preguntas a incluir en el cuestionario, realizaron tres versiones del mismo diferenciadas únicamente en el número de páginas y en los cambios necesarios para ello: un cuestionario tiene tres páginas, otro seis y otro nueve. $\mathrm{Al}$ analizar la tasa de respuesta de cada uno, el de nueve páginas consigue una respuesta mayor, provocada sin duda por los aspectos visuales del mismo (Champion y Sear, 1969). Similares resultados son expuestos por Dillman, Sinclair y Clark (1993) en un experimento realizado con el censo de 1990. Estos autores comparan la tasa de respuesta de distintos tipos de cuestionarios censales obteniendo unas tasas de respuesta del 66,8 por 100 para el cuestionario de ocho páginas, 71,4 por 100 el de una página y 70,9 por 100 el que tienen menos de una página. La influencia del tamaño de cuestionario es significativa, pero nosotros nos preguntamos hasta qué punto una diferencia del 4,6 por 100 proporciona suficiente evidencia para reducir un cuestionario de ocho páginas a una, siempre que el número y naturaleza de las preguntas se correspondan con el objeto de estudio. En una de las últimas investigaciones realizadas sobre este tema, Scott y Sechrest (1993) encuentran una escasa diferencia ( 2 por 100 ) en la tasa de respuesta entre dos cuestionarios, uno de ellos tres

${ }^{20}$ Scott (1961); Brown (1965); Champion y Sear (1969); Berdie (1973); Linsky (1975); Roscoe, Lang y Sheth (1975); Heberlein y Baumgartner (1978); Childers y Ferrel (1979); Yu y Coper (1983); Fox, Crask y Kim (1988). 
veces más largo que el otro. No obstante, la calidad de la respuesta del cuestionario más largo se deteriora enormemente en las últimas preguntas.

Algunas investigaciones han restado importancia a la longitud del cuestionario considerando que la temática del mismo y el interés de los entrevistados es lo realmente importante, puesto que la percepción del paso del tiempo es distinta cuando alguien está haciendo algo en lo que está interesado (Boek y Lade, 1963; Heberlein y Baumgartner, 1978). Suponemos que esta afirmación tiene poco interés para los investigadores interesados en el tema puesto que el tema del cuestionario es algo que no puede ser modificado, con lo cual no puede incrementarse la colaboración cambiando el tema del mismo, mientras sí puede hacerse modificando el formato del mismo.

Conforme avanzamos en nuestra exposición hacia factores más específicos de la encuesta postal, nos encontramos con menos investigaciones y con resultados sustentados en un menor número de estudios. Desearíamos incidir en una idea expuesta anteriormente: el hecho que algunos resultados se han obtenido de poblaciones especializadas hace temer sobre la generalización de los mismos. No estamos diciendo que no puedan ser generalizables, sino que no estamos totalmente seguros de tal generalización. En esta parte se ha dado cuenta de la influencia del tamaño del cuestionario en cuanto a su formato, la importancia del diseño de portada y contraportada, la mejora producida por los cuestionarios de colores, y la escasa influencia del diseño en cuanto a calidad de impresión.

\section{INSTITUCIÓN RESPONSABLE DE LA INVESTIGACIÓN}

La peculiaridad que tiene este último factor es la nula influencia que el investigador tiene sobre el mismo, ya que es muy difícil cambiar la institución a la que se pertenece y la imagen de la misma. La incidencia de la institución responsable fue analizada en primer lugar por Scott (1961) en Gran Bretaña comparando las tasas de respuesta de un mismo cuestionario enviado por tres organismos diferentes: una oficina del gobierno, una universidad (London School of Economics) y una institución privada. Aunque las tasas de respuesta no experimentaron grandes variaciones $(93,89$ y 90 por 100 , respectivamente), la significación estadística de éstas le llevaron a afirmar la existencia de una relación entre el organismo convocante y la tasa de respuesta. Esta opinión es compartida por otros autores como Roeher (1963), Vocino (1977), Labrecque (1978), Dillman (1978), Jones y Lang (1980).

En una investigación que analiza la influencia de la prenotificación en la población general, Brunner y Carroll (1988) encuentran que una prenotificación enviada por la Universidad de Maryland aumenta la tasa de respuesta un 20 por 100 , mientras que el envío por una institución privada hace que ésta disminuya ligeramente, aunque no de modo significativo. Al analizar únicamente la submuestra que ha recibido la prenotificación, la universidad consigue una tasa de 
respuesta del 72,5 por 100 , mientras que la institución privada llega al 46,1 por 100. El análisis de cómo afecta la prenotificación a la respuesta de dos preguntas sensibles (sobre ahorros y salarios) señala que la universidad genera una mayor tasa de colaboración, aunque las diferencias no llegan a ser significativas.

Estudios realizados por Cox, Anderson y Fulcher (1974), Peterson (1975), Scott (1961) y Vocino (1977) señalan que la influencia de la institución es distinta según el tipo de población analizada: así, los profesores y el público en general responden mejor a una universidad, mientras que los miembros de sociedades y empresas responden mejor a los requerimientos de éstas que a la solicitud de una universidad.

Aunque algunos trabajos como los realizados por Watson (1965) y Friedman-San Augustine (1979) no encuentran tasas de respuesta distintas según el organismo que remita la investigación, concluimos señalando la gran importancia de la institución que promueve la investigación, fundamentalmente cuando se trata de universidades u organismos públicos. El hecho que una institución estatal, una universidad, etc., esté realizando una investigación aumentará la probabilidad de cooperación. Para finalizar, y pese a la importancia de este factor, el problema es que nada podemos hacer para modificarlo, como ya se ha expuesto anteriormente.

\section{CONCLUSIÓN}

Comenzábamos este artículo señalando la importancia de la encuesta en la investigación social, al tiempo que hemos detectado un desajuste entre esta importancia y el escaso número de publicaciones existentes en nuestro país sobre las técnicas de mejora de la calidad de los datos recogidos, la incidencia de las nuevas innovaciones, etc. No insistiríamos en este aspecto de no ser por el alarmante descenso de la cooperación de la población a entrevistar. No obstante, esta disminución varía según el método de recogida de datos utilizado: una de las últimas investigaciones sobre el tema obtenía una tasa de respuesta en las entrevistas personales del 51 por 100 , porcentaje que aumentaba al 66 por 100 en el caso de las telefónicas y al 68 por 100 en el caso de las postales (DeLeeuw, 1996: 449). No conocemos ningún estudio sobre el tema en nuestro país, si bien estudiosos en la materia nos hablan de una tasa de cooperación similar, aunque algo más alta, en la encuesta personal. Esta reflexión nos anima a plantear unas preguntas fundamentales: si la tasa de respuesta de la encuesta personal es tan baja, ¿cuál es la razón por la que no se buscan alternativas que mejoren este método? ¿Que tipo de investigación estamos realizando si sólo obtenemos las respuestas de un determinado sector de la población?

Este trabajo ha pretendido dar respuesta a estas preguntas proponiendo un método de recogida de datos muy poco utilizado en el entorno europeo. Planteábamos como hipótesis que la tasa de respuesta y la calidad de los datos recogidos por encuestas postales es semejante a los recogidos por la encuesta personal y telefónica. A excepción de las investigaciones que necesitan un método rápido 
de recogida de datos y otras situaciones específicas que expondremos al final de este apartado, el resto de trabajos supone un área excelente para la utilización de encuestas postales, dado que la mayoría de las investigaciones buscan conocer situaciones y problemas sociales en los cuales la rapidez en la obtención de los datos no es un aspecto crucial.

A lo largo del artículo se ha podido comprobar cómo existe un gran acuerdo en que el factor que más incide en las tasas de respuesta es el aumento del número de contactos tanto en forma de prenotificación como de recuerdo, bien es verdad que los recordatorios producen un aumento en la respuesta mayor que la prenotificación. El segundo factor más influyente es el uso de incentivos, aunque su efecto está condicionado según el modo de recibirlos y el tipo del incentivo: son más efectivos los incentivos monetarios que los no monetarios, y producen mayor tasa de respuesta los que se envían antes de contestar que los enviados tras la respuesta del cuestionario. Personificar la correspondencia y el nombre de la institución que organiza la investigación son los elementos que influyen en tercer lugar, si bien este último es un elemento no tan importante ya que poco podemos hacer para modificarlo. El resto de los factores analizados (franqueo postal, anonimato, carta de presentación, cuestionario) tienen una influencia menor. Obviamos el hecho de incluir un sobre franqueado para la respuesta por parecer demasiado evidente y tener la certeza de que todo aquel que piense realizar un trabajo de este tipo incluirá un sobre y un sello para facilitar la respuesta.

En síntesis, consideramos que la utilización de encuestas postales nos permite obtener datos con una calidad similar a los recogidos mediante encuestas personales y telefónicas, aunque para ello sea necesario utilizar determinadas estrategias que han sido expuestas en las páginas anteriores. Además, la encuesta postal nos permite recoger estos datos reduciendo tremendamente el costo del trabajo de campo; aspectos que nos llevan a considerar la encuesta postal como uno de los métodos de recogida de datos con mayor futuro.

Para finalizar, nos gustaría realizar una breve exposición de las situaciones donde no es aconsejable la utilización de encuestas postales; situaciones que están más relacionadas con las "limitaciones del medio" que con las temáticas a analizar: las encuestas postales han sido utilizadas con éxito en investigaciones sobre actitudes y comportamientos, actitudes hacia emigrantes, racismo, medio ambiente, conductas sexuales, etc. Incluso existen estudios realizados con encuestas postales que han predicho resultados electorales con notable éxito (Visser, 1996). Como decíamos, los campos de no aplicación de la encuesta postal están relacionados con los inconvenientes del medio de recogida de datos: lentitud en las respuestas, incertidumbre sobre la identidad del entrevistado, imposibilidad para aislar el efecto de terceras personas en las respuestas, no apropiado para cuestionarios complejos o difíciles de contestar, no apropiado para personas mayores o con bajo nivel educativo, imposibilidad de ayuda al entrevistado, etc. Aspectos que están siendo desarrollados por el autor en un nuevo trabajo sobre el tema. 


\section{BIBLIOGRAFÍA}

Alos, J. S. (1996): "Industria de los Estudios de Mercado en España 1995", Investigación y Marketing, vol. 53, pp. 64-68.

Anidrfasen, A. R. (1970): "Personalizaning Mail Questionnaire Correspondence", Public Opinion Quarterly, vol. 34, pp. 272-277.

Armstrong, J. S., y Lusk, E. J. (1987): «Return Postage in Mail Surveys", Public Opinion Quarterly, vol. 51, pp. 233-248.

BallEy, K. D. (1978): Methods of Social Research, Nueva York: Free Press.

BERIIE, D. R. (1973): "Questionnaire Length and Response Rate", Journal of Applied Psychology, vol. 58 , pp. $278-280$.

Bl.ack, J. A., y Champion, D. J. (1976): Methods and Issues in Social Research, Nueva York: Wiley.

Bokk, W., y LaDe, J. (1963): "A Test of the Usefulness of the Postcard Technique in a Mail Questionnaire Study", Public Opinion Quarterly, vol. 27, pp. 299-302.

BrenNAN, M., y HoEk, J. (1992): "The Behavior of Responders, Nonresponders and Refusal Across Mail Surveys", Public Opinion Quarterly, vol. 56, pp. 530-535.

BROWN, M. L. (1965): "The Use of a Postcard Query in Mail Surveys», Public Opinion Quarterly, vol. 29, pp. 635-637.

Bosh, J. L., y ToRRENTE, D. (1993): Encuestas Telefónicas y por Correo, Madrid: CIS.

Brunner, G. A., y Carroll, S. J. (1988): "The Effect of Prior Notification on the Refusal Rate in Fixed Address Surveys", Journal of Advertising Research, vol. 9, pp. 42-44.

BURCHELl, B., y MARSH, C. (1992): "The Effect of Questionnaire Length on Survey Response", Quality and Quantity, vol. 26, pp. 233-244.

CARpinTER, E. W. (1974): “Personalization in Mail Surveys of the General Public: a Reassessment in Light of the Innovations", Public Opinion Quarterly, vol. 38, pp. 4-15.

CARTWRIGHT, A., y WARI), A. W. M. (1968): "Variations in General Practitioner's Response to Postal Questionnaires", British Journal of Preventive and Social Medicine, vol. 22, pp. 199. 205.

Champion, D. J., y Sear, A. M. (1969): "Questionnaire Response Rates: a Methodological Analysis», Social Forces, vol. 47, pp. 335-339.

Childers, T. L., y Ferrei, O. C. (1979): «Response Rates and Questionnaire Length in Mail Surveys", Journal of Marketing Research, vol. 16, pp. 429-431.

CHurch, A. H. (1993): «Estimating the Effect of Incentives on Mail Surveys Response Rates: A Meta-Analysis", Public Opinion Quarterly, vol. 57, pp. 62-79.

Cox, E. P.; Anderson, W. T., y Fulcher, D. G. (1974): «Reapprasing Mail Survays Response Rates", Journal of Marketing Research, vol. 11, pp. 413-417.

Crittenden, W.; CritTendin, V., y Hawes, J. (1985): "Examining the Effects of Questionnaire Color and Print Front on Mail Survey Response Rates», Akron Business and Economic Review, vol. 16, pp. 51-56.

DeLeEUW, E. D., y Hox, J. J. (1988): "The Effects of Response-Stimulating Factors on Response Rates and Data Quality in Mail Surveys», Journal of Official Statistics, vol. 4, pp. 241-249.

DeLeeuw, E. D., et al. (1996): "The Influence of Data Collection Methods on Structural Models", Sociological Methods and Research, vol. 24, pp. 443-472.

DEMAIO, T. J. (1984): "Social Desirability and Survey Measurement: A review», en C. T. TURNER y E. MARTIN, Surveying Subjective Phenomena, Nueva York: Russel Sabe Fundation, pP. $257-282$.

Diliman, D. A. (1978): Mail and Telephone Surveys, Nueva York: Willey.

- (1983): "Mail and Other Self-Administered Questionnaires", en Rossi, Wright y Anderson (eds.), Handbook of Survey Research, Academic-Press, pp. 359-377.

- (1991): "The Design and Administration of Mail Surveys", Annual Review of Sociology, vol. 17 , pp. 225-249. 
Diliman, D. A.; Carpent'ir, E.; Christenson, J., y Brooks, R. (1974): «Increasing Mail Questionnaire Response: a Four State Comparison», American Sociological Review, vol. 39, pp. 744-756.

Diliman, D. A.; Ciark, J. R., y Sinclaik, M. D. (1995): "How Prenotice Letters, Stamped Return Envelopes and Reminder Postcards Affect Mailback Response Rates for Census Questionnaires", Survey Methodology, vol. 21, pp. 159-165.

Dillman, D. A.; Dillman, J. J., y Makfl.A, C. J. (1984): "The importance of Adhering to Details of the Total Design Method (TDM) for Mail Surveys", en Daniel C. Lockart (ed.), New Directions for Program Evaluation, San Francisco: Jossey-Bass, pp. 49-64.

DIILMAN, D. A.; SINCLAIR, M. D., y Cl.ARK, J. R. (1993): "Effects of Questionnaire Length, Respondent-Friendly Design, and a Difficult Questions on Response Rates for OccupantAddressed Census Mail Surveys", Public Opinion Quarterly, vol. 57, pp. $289-304$.

Diliman, D. A.; Sincer, E; Ciark, J. R., y Trea't, J. B. (1996): «Effects of Benefits Appeals, Mandatory Appeals, and Variations in Statements of Confidentiality on Completion Rates for Census Questionnaires», Public Opinion Quarterly, vol. 60, pp. 376-389.

DILImAN, D. A.; WLST, K. K., y Cl.ARK, J. R. (1994): "Influence of an Invitation to Answer by Telephone on Response to Census Questionnaires", Public Opinion Quarterly, vol. 58, pp. $557-568$.

DIILMAN, D. A., y FRFY, J. H. (1974): “Contribution of Personalization to Mail Questionnaire Response as an Element of a Previously Tested Method", Journal of Applied Psychology, vol. 59, pp. 297-301.

Dillman, D. A., y Mooke, D. E. (1983): Improving Response to Mail Surveys: Results From Five Experiments, Unpublished Manuscript, Pullman, Washington: Washington State University.

Downs, P. E., y Ktr, J. R. (1986): «Recent Evidence on the Relationship Between Anonimity and Response Variables for Mail Surveys", en Journal of the Academy of Marketing Science, vol. 14, pp. $72-82$.

ECKLANI), B. K. (1965): «Effects of Prodding to Increase Mail Back Returns», Journal of Applied Psychology, vol. 49, pp. 165-169.

Eki>os, P. L., y Mocian, A. J. (1983): Professional Mail Surveys, Malabar: FL.

FARIA, A. J.; Dickinson, J. R,, y FlliplC, T. V. (1990): "The Effect of Telephone versus Letter Prenotification on Mail Survey Response Rate", Journal of the Market Research Society, vol. 32, pp. 551-568.

Fox, R. J.; CrAsk, M. R., y KIM, J. (1988): "Mail Survey Response Rate (A Meta-analysis of Selected Techniques for Inducing Response)", Public Opinion Quarterly, vol. 52, pp. 467-491.

Frey, J. H. (1989): Survey Research by Telephone, Newbury Park, CA: Sage.

Frieidman, H. H., y SAN Augustine, A. J. (1979): "The Effects of a Monetary Incentive and the Ethnicity of the Sponsor's Signature on the Rate and Quality of Response to a Mail Survey", Journal of the Academy of Marketing Science, vol. 17, pp. 95-100.

Furse, D. H., y STFWARD, D. W. (1982): «Monetary Incentives Versus Promised Contribution to Charity: New Evidence on Mail Survey Response", Journal of Marketing Research, vol. 19, pp. 375-380.

FuTRli.l, C. M., y Hist, R. T. (1982): "The Effects of Anonymity and a Same Day Deadline on the response Rate to Mail Surveys", European Research, vol. 10, pp. 171-175.

GoYDER, J. (1982): "Further Evidence on Factors Affecting Response Rates to Mailed Questionnaires", American Sociological Review, vol. 47, pp. 550-553.

- (1987): The Silent Minority, Cambridge: Polity Press.

GRtMBowsk! (1985): "Survey Questionnaire Salience", American Journal of Public Health, vol. 75, pp. 1350-60.

Grovfs, R. M.; Cialidni, R. B., y COUPER, M. P. (1992): "Understanding the Desicion to Participate in a Survey", Public Opinion Quarterly, vol. 56, pp. 475-495.

Guilahorn, J. T., y Gullahorn, J. F. (1963): "An Investigation of the Effects of Three Factors on Response to Mail Questionnaires", Public Opinion Quarterly, vol. 27, pp. 276-281.

GullaHORN, J. T., y GULLAHORN, J. F. (1959): "Increasing Returns From Non-respondents", Public Opinion Quarterly, vol. 23, pp. 119-121. 
Hansen, R. A., y Robinsun, L. M. (1980): "Testing the Effectiveness of Alternative Foot-inthe-door Manipulations", Journal of Marketing Research, vol. 17, pp. 359-364.

Heberlein, T., y BAUMGAR'INER, R. (1978): "Factors affecting response rates to mailed questionnaires: A quantitative analysis of the published literature", American Sociological Review, vol. 43, 447-462.

Henley, J. R. (1976): «Response Rate to Mail Questionnaires with a Return Deadline», Public Opinión Quarterly, vol. 40, pp. 374-375.

Hopkins, K. D., y Gullickson, A. R. (1992): "Response Rates in Surveys Research: A MetaAnalysis of the Effect of Monetary Gratuties», Journal of Experimental Education, vol. 61, pp. $52-62$.

Hornik, J. (1981): "Time Cue and Time Perception Effect on Response to Mail Surveys", Journal of Marketing Research, vol. 18, pp. 243-248.

Houston, M., y Nevin, J. R. (1977): "The Effects of Source and Appeal on Mail Survey Response Patterns", Journal of Marketing Research, vol. 14, pp. 374-378.

Hox, J. J., y DeLeevw, E. (1992): “A Comparison of Nonresponse in Mail, Telephone, and Face-to-face Surveys", Working-Papers, Universitei van Amsterdam, núm. 49.

JAMFS, J. M., y BOLSTEIN, R. (1992): «Large Monetary Incentives and Their Effect on Mail Survey Response Rates", Public Opinion Quarterly, vol. 56, pp. 442-453.

JANSEN, J. H. (1985): «Effect of Questionnaire Layout And Size And Issue-Involvement on Response Rates in Mail Surveys", Perceptual and Motor Skills, vol. 61, pp. 139-142.

Janssens, D., y Pessemier, E. A. (1980): Response Rates in Mail Surveys: A Review and Survey, Institute for Research in the Behavioral, Economic, and Management Sciences, Purdue University, Indiana, Paper no. 714.

Jenkins, C. R., y Diliman, D. A. (1996): "Towards a Theory of Self-Administered Questionnaire Design», en LYBerg et al., Survey Measurement and Process Quality, Nueva York: John Wiley \& Sons.

Jobber, D. (1986): "Improving Response Rates in Industrial Mail Surveys», Industrial Marketing Management, vol. 15, pp. 183-195.

Jobber, D.; Alien, N., y OAKI.ANi), J. (1985): "The impact of Telephone Notification Strategies on Response to an Industrial Mail survey", International Journal of Research in Marketing, vol. 2, pp. 291-296.

JONES, W. H., y LANG, J. R. (1980): "Sample Composition bias and Response biass in a Mail Survey: A comparison of Inducement Methods", Journal of Marketing Research, vol. 17, pp. $69-76$.

JoNES, W., y LiNDA, G. (1978): "Multiple Criteria Effects in a Mail Survey Experiment», Journal of Marketing Research, vol. 15, pp. 280-284.

Johnson, T. P.; Parsons, J. A.; Warnecke, R. B., y Kaluny, A. D. (1993): «Dimensions of Mail Questionnaires and Response Quality", en Sociological Focus, vol. 26, pp. 271-274.

Kviz, F. J. (1977): "Towards a Standard Definition of Response Rate», Public Opinion Quarterly, vol. 41, pp. 265-267.

LABRECQUe, D. P. (1978): "A Response Rate Experiment Using Mail Questionnaires", Journal of Marketing, vol. 42, pp. 82-83.

LINSKY, A. S. (1975): «Stimulating Responses to Mailed Questionnaires: A Review», Public Opinion Quarterly, vol. 39, pp. 82-101.

LiNSKY, A. S. (1965): "A Factorial Experiment to Inducing Responses to a Mail Questionnaire», Sociolgy and Social Research, vol. 39, pp. 183-189.

LONGWORTH, D. S. (1953): "Use of a Mail Questionnaire», American Sociological Review, vol. 18 , pp. $310-313$.

MATTESON, M. (1974): "Type of Transmittal Letter and Questionnaire Color as Two Variables Influencing Response Rates in a Mail Survey", Journal of Applied Psychology, vol. 59, pp. 535-536.

MASON, W. S., et al. (1961): “An Experimental Study of Factors Affectting Response Rate to a Mail Survey of Beginning Teachers", Public Opinion Quarterly, vol. 25, pp. 296-299. 
MoCrohan, K. F., y Lowe, L. S. (1981): «A Cost Benefit Approach to Postage Used on Mail Questionnaires", Journal of Marketing, vol. 45, pp. 130-133.

MCDANifi, S. W., y RAO, C. P. (1981): "An Investigation of Respondent Anonymity's Effect on Mail Questionnaire Response Rate and Quality», Journal of the Market Research Society, vol. 23, pp. 150-160.

Mryers, L. S., y Grossfin, N. E. (1974): Behavioral Research: Theory, Procedure and Design, San Francisco: Freeman.

Nenerhof, A. J. (1983): "The Effects of Repetition and Consistency of Personalization Treatment of Response Rates in Mail Surveys", Social Science Research, vol. 12, pp. 1-9.

- (1985a): "A Comparison of European and North American Response Patterns in Mail Survey", Journal of the Market Research Society, vol. 27, pp. 55-63.

- (19856): "A Survey on suicide: Using a Mail Survey to Study a highly Threatening Topic", Quality and Quantity, vol. 19, pp. 293-302.

- (1988): "Effects of a Final Telephone Reminder and Questionnaire Cover Design in Mail Surveys", Social Science Research, vol. 17, pp. 353-361.

PAIIWODA, S. J. (1981): "Using a Mail Questionnaire as a Research Tool", Quarterly Review of Marketing, vol. 6, pp. 9-14.

PEIIERSON, R. A. (1975): “An Experimental Investigation of Mail Survey Responses», Journal of Business Research, vol. 3, pp. 199-208.

Powtrs, D. E., y Al.DeRman, D. L. (1982): «Feedback as an Incentive for Responding to a Mail Questionnaire», Research in Higher Education, vol. 17, pp. 207-211.

Pressiey, M. M., y Tuller, W. L. (1977): «A Factor Interactive Investigation of Mail Survey Response Rates from a Commercial Population», Journal of Marketing Research, vol. 14, pp. 108-111.

Rorfris, R. E.; MCCRory, O. F., y FORTHOFer, R. N. (1978): "Further Evidence to Stimulate Responses to a Mail Surveys», Public Opinion Quarterly, vol. 42, pp. 407-410.

Robertson, D. H., y Beilifnger, D. (1978): "A New Method of Increasing Mail Survey Responses: Contributions to Charity", Journal of Marketing Research, vol. 15, pp. 632-633.

ROBin, D. P., y WaITERS, C. O. (1976): "The Effect on Return Rates of Messages Explaining Monetary Incentives in Mail Questionnaire Studies", Journal of Business Comunication, vol. 13 , pp. $49-53$.

Rofhte, G. A. (1963): «Efectiveness Techniques in Increasing Response to Mailed Questionnaires", Public Opinion Quarterly, vol. 27, pp. 299-302.

Roscoe, A. M.; LANG, D., y SHE1H, J. N. (1975): "Follow-up Methods, Questionnaire Length and Market Differences in Mail Surveys", Journal of Marketing, vol. 39, pp. 20-27.

Scott, A. G., y Sechrest, L. (1993): "Survey Research and Response Bias", Proceedings of the Section on Survey Research Methods, American Statistical Association, vol. I, pp. 238-243.

SCOIT, C. (1961): "Research on Mail Surveys", Journal of the Royal Statistical Society, pp. 143195.

SIMON, R. E. (1967): "Response to Personal and Form Letters in Mail Surveys», Journal of Advertising Research, vol. 7, pp. 28-30.

SinciER, E. (1978): «Informed Consent: Consecuences for Response Rate and Response Quality in Social Surveys», American Sociological Review, vol. 43, pp. 144-162.

SMith, T. W. (1995): "Trends in Nonresponse Rates», International Journal of Public Opinion Research, vol. 7, pp. 157-171.

STrvens, R. E. (1974): "Does Precoding Mail Questionnaires Affect Response Rates?", Public Opinion Quarterly, vol. 38, pp. 621-622.

Sucityama, M. (1992): "Responses and Non-responses", en Lebart (ed.), Quality of Information in Sample Surveys, París: Dunod, pp. 227-239.

Vlsser, P. S., et al. (1996): "Mail Surveys for Election Forecasting?", Public Opinion Quarterly, vol. 60, pp. 181-277.

Vocino, T. (1977): "Three Variables in Stimulating Response to Mail Questionnaires", Journal of Marketing, vol. 41, pp. $76-77$. 
Yammarino, F. J.; Skinner, S. J., y Childorens, T. L. (1991): "Understanding Mail Survey Response Behaviour: A Meta-analysis», Public Opinion Quarterly, vol. 55, pp. 613-639.

YU, J., y COOPER, H. (1983): "A Quantitative Review of Research Design Effects on Response Rates to Questionnaires", Journal of Marketing Research, vol. 20, pp. 26-44.

Watson, J. J. (1965): "Improving the Response Rate in Mail Research", Journal of Advertising Research, vol. 5, pp. 45-50.

WynN, G. W., y MCDANIFl, S. W. (1985): "The Effect of Alternative Foot-in-the-Door Manipulations on Mailed Questionnaire Response Rate and Quality", Journal of Market Research Society, vol. 27, pp. 15-26.

\begin{abstract}
This paper sets out to analyse the factors which contribute to the improved quality and response rate vis-à-vis postal surveys. Research in this area was largely encouraged by the fact that while participation in face-to-face and telephone interviews has been falling in recent years, participation in postal surveys has remained stable. Accordingly, this paper summarises the factors which influence the efficiency of postal surveys, its main hypothesis being that the rate of response and the quality of the data collected through postal surveys is similar - and sometimes superior - to the data compiled in one-to-one and telephone surveys, and at a substantially lower cost.
\end{abstract}

